\title{
EFFECTS OF A NEW PREBIOTIC MIXTURE ON FAECAL GUT MICROBIOTA AND THE MUCOSAL IMMUNE SYSTEM IN HEALTHY INFANTS
}

\author{
R. Martin ${ }^{1}$, M. Friedt ${ }^{2}$, A. Buco ${ }^{1}$, E. Zoetendal ${ }^{3}$, K. Ben Amor ${ }^{4}$, J. Knol ${ }^{1}$, C.P. Braegger ${ }^{5}$, MIPS Study \\ Group \\ ${ }^{1}$ Danone Research - Centre for Specialised Nutrition, Wageningen, The Netherlands, ${ }^{2}$ Division of \\ Gastroenterology, Hepatology and Nutrition, Klinik für Allgemeine Pädiatrie-Klinikum der Heinrich-Heine \\ Universität Düsseldorf, Dusseldorf, Germany, ${ }^{3}$ Microbiology, Wageningen University, Wageningen, The \\ Netherlands, ${ }^{4}$ Danone Research-Asia Pacific, Singapore, Singapore, ${ }^{5}$ Division of Paediatric \\ Gastroenterology and Nutrition, University Children's Hospital, Zurich, Switzerland
}

Background and aims: It has recently been shown that a specific mixture of neutral (scGOS/lcFOS) and acidic oligosaccharides (pAOS) can prevent the onset of atopic dermatitis (AD) in healthy infants (Gruber et al., 2010). The intestinal microbiota plays a crucial role in the maturation of the gut, therefore we investigated the effect of this mixture on gut microbiota parameters and faecal SIgA.

Methods: In a randomised double-blind placebo controlled multi-centre study, 1187 healthy term infants received either a formula supplemented with scGOS/lcFOS/pAOS (prebiotic), a standard formula (control) or breast milk (reference). Microbial composition, short chain fatty acids (SCFA), lactate, $\mathrm{pH}$ and SIgA were analysed in 239 infants at 2, 4 and 12 months. In a subset, the microbial diversity was assessed by phylogenetic arrays.

Results: The microbiota composition of prebiotic formula fed infants resembles that of breastfed infants with significantly lower $\mathrm{pH}$; higher proportion of acetate and lower proportion of butyrate, propionate; and the branched SCFA when compared to the control group. Faecal SIgA levels in the prebiotic group were significantly higher than the control group at 4 and 12 months and similar to the breasted infant at both time points. The array data show the microbiota dynamics and allows a more detailed comparison in the first year of life.

Conclusions: These findings show an infant formula supplemented with scGOS/lcFOS/pAOS has beneficial effects on $\mathrm{AD}$ prevention, which can be linked to an effect on faecal microbiota composition; metabolic activity and the mucosal immune system comparable to human milk. 